CERN LIBRARIES, GENEVA

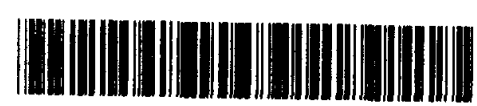

CERN-AT-95-25
CERN AT/95-25 (MA)

LHC Note 340

$$
\text { fue } 5547
$$

Power Tests of Single and Twin Aperture Superconducting Dipole Models for LHC

L. Walckiers, D. Leroy, L. Oberli, J. Ostler, F. Rodriguez-Mateos, N. Siegel, A. Siemko

High field superconducting magnets working at superfluid helium temperature are requested for the $7 \mathrm{TeV}$ LHC accelerator. Several single and twin aperture $1 \mathrm{~m}$ long dipole models were measured to compare the differences in behaviour due to design and fabrication. They are shown to be valid models to predict the behaviour of full length magnets. The analysis of the numerous data collected during the performance tests of these magnets allows the proposal of some guidelines to understand the causes of the training quenches. Short sample measurements of both single strands and cables are compared with ultimate field values obtained on the magnets. 


\title{
Power Tests of Single and Twin Aperture Superconducting Dipole Models for LHC
}

\author{
L. Walckiers, D. Leroy, L. Oberli, J. Ostler, F. Rodriguez Mateos, N. Siegel, A. Siemko, \\ CERN, European Organization for Nuclear Research. $\mathrm{CH}-1211$ Geneva 23
}

\begin{abstract}
High field superconducting magnets working at superfluid helium temperature are requested for the $7 \mathrm{TeV}$ LHC accelerator. Several single and twin aperture $1 \mathrm{~m}$ long dipole models were measured to compare the differences in behaviour due to design and fabrication. They are shown to be valid models to predict the behaviour of full length magnets. The analysis of the numerous data collected during the performance tests of these magnets allows the proposal of some guidelines to understand the causes of the training quenches. Short sample measurements of both single strands and cables are compared with ultimate field values obtained on the magnets.
\end{abstract}

\section{INTRODUCTION}

CERN will operate the $\mathrm{LHC}$, a $7 \mathrm{TeV}$ proton-proton collider in the middle of the next decade [1]. Twin-aperture $\mathrm{NbTi}$ superconducting dipole magnets are demanding elements of this particle accelerator. They will reach the specified field of $8.36 \mathrm{~T}$ with a Rutherford type cable conductor immersed in a helium bath of $1.9 \mathrm{~K}$ at atmospheric pressure.

A first generation of short dipole models was designed at the end of the nineteen-eighties to study the feasibility of these magnets [2]. Six single-aperture and seven twinaperture model magnets with a bore diameter of $50 \mathrm{~mm}$ were tested. Most have a $17 \mathrm{~mm}$ wide cable, three magnets manufactured by the KEK laboratory have a cable width of $15 \mathrm{~mm}$ with a large keystoning angle [3], the MBTRA magnet was wound with the $12 \mathrm{~mm}$ SSC cable [4]. Four 10 $\mathrm{m}$ long magnets based on the $17 \mathrm{~mm}$ cable version have been tested in the last 15 months.

To date, 45 cold tests of short models have been performed. Specific studies have been presented in separate publications [5], [6].

Premature quenches are a serious problem for magnets that have to work at $87 \%$ of the superconductor limit. These quenches are provoked by movements of the conductors that are submitted to unprecedented high Lorenz forces for accelerator dipole magnets. The behaviour of these magnets with respect to thermal cycles or long period at room temperature is also of practical importance.

Before testing magnets based on the new parameters (15-mm-wide cable, 56-mm bore aperture), it is worthwhile to collect further evidence that $1-\mathrm{m}$-long dipoles with single and twin apertures are relevant models to validate the design of the $14 \mathrm{~m}$ full length magnets of the LHC.

\section{The Critical Current Limit of the Magnets}

Critical currents were systematically measured at $4.3 \mathrm{~K}$ on single strands extracted from the cables used in the model magnets. Measurements have also been performed at $1.8 \mathrm{~K}$ to $2.0 \mathrm{~K}$ to deduce the effect of the reduction of temperature for each supplier [7]. Cables were also measured at BNL at $4.2 \mathrm{~K}$ with the dipole field perpendicular to the broad face. The critical currents measured on the cables compare well with the sum of the critical currents of the strands extracted from the cable, if the self field is taken into account in the cable measurements. The validity of that rule shows that the quench starts in one strand of the cable.

All short and long models tested so far were brought to their conductor limit at $4.3 \mathrm{~K}$. Some $1-\mathrm{m}$-long models have reached their limit in superfluid temperatures by leaving the bath slowly warming up at constant current. It was checked in both cases that the resulting quenches start repeatedly in the high field region of the straight part.

The conductors' limits predicted by short sample measurements are compared in Table I with values measured on the magnets in both atmospheric boiling and superfluid helium. To normalize the results from the magnets at $4.35 \mathrm{~K}$ and $2.0 \mathrm{~K}$, linear corrections with respect to the real temperature are applied. The predictions are valid within $2 \%$ at both temperatures.

\section{TABLEI}

MAXIMUM FELDS EXPECTED FROM SHORT SAMPLE LMMT MEASUREMENTS COMPARED WITH THE FIELDS OBTAINED ON $1 \mathrm{~m}$ LONG AND $10 \mathrm{~m}$ LONG MODEL MAGNETS

\begin{tabular}{|c|c|c|c|c|c|c|}
\hline \multirow[t]{2}{*}{ Magnet } & \multicolumn{2}{|c|}{ Magnets limit at } & \multicolumn{2}{|c|}{ Short sample timit } & \multicolumn{2}{|c|}{ Difference $[\boldsymbol{\phi}]$} \\
\hline & $4.35 \mathrm{~K}$ & $2.0 \mathrm{~K}$ & $4.35 \mathrm{~K}$ & $2.0 \mathrm{~K}$ & $4.35 \mathrm{~K}$ & $2.0 \mathrm{~K}$ \\
\hline \multicolumn{7}{|l|}{$1 \mathrm{~m}$ long } \\
\hline $\begin{array}{l}\text { MTAIA } \\
\text { MTAIE } \\
\text { MTAIJ } \\
\text { MTA3C } \\
\text { MBTRA }\end{array}$ & $\begin{array}{l}7.9 \\
7.79 \\
7.75 \\
8.13 \\
7.23\end{array}$ & $\begin{array}{r}10.20 \\
10.60 \\
9.65\end{array}$ & $\begin{array}{l}7.82 \\
7.69 \\
7.83 \\
7.97 \\
7.12\end{array}$ & $\begin{array}{r}10.22 \\
10.40 \\
9.31\end{array}$ & $\begin{array}{l}1 \\
1.3 \\
-1 \\
2 \\
1.5\end{array}$ & $\begin{array}{c}-0.2 \\
2 \\
3.6\end{array}$ \\
\hline \multicolumn{7}{|l|}{$10 \mathrm{~m}$ long } \\
\hline $\begin{array}{l}\text { MTPIAI } \\
\text { MTPIAZ } \\
\text { MTPIA3 } \\
\text { MTPINI }\end{array}$ & $\begin{array}{l}7.96 \\
7.80 \\
7.87 \\
7.80 \\
\end{array}$ & & $\begin{array}{l}7.91 \\
7.73 \\
7.8 \\
7.78 \\
\end{array}$ & $\begin{array}{l}10.23 \\
10.06 \\
10.12 \\
10.22 \\
\end{array}$ & $\begin{array}{l}0.6 \\
0.9 \\
0.9 \\
0.3 \\
\end{array}$ & \\
\hline
\end{tabular}


A relationship can also be deduced in order to scale from the limit at $4.35 \mathrm{~K}$ to that at $2.0 \mathrm{~K}$. A constant enhancement of $1.31 \pm 1.5 \%$ is measured whatever the magnet or cable considered. This relationship is very useful to deduce the limit of a magnet at $2 \mathrm{~K}$ if it cannot be directly measured.

\section{Measured Training Performances}

Recent studies made during the power tests of both $1 \mathrm{~m}$ and $10 \mathrm{~m}$ long model magnets have clarified our knowledge about what influences the level of premature quenches.

\section{A. The Mechanism of Premature Quenches}

Numerous spikes, signatures of sudden movements of conductors that release energy, are always observed mainly at the first powering of a winding. A small fraction of these spikes release sufficient energy to bring the conductor above the critical temperature and provoke a quench. The resulting wave that propagates in the winding can provoke a quench in a different location if that location is more susceptible to dissipative movements.

\section{B. Changing the Current Cycling Conditions}

Different current ramp rates or cycling conditions could either decrease the frequency of the spikes, or provoke the related energy releases at a lower current, therefore influencing the training.

Going up and down with fixed current intervals to approach by steps the quench level was systematically tried on every second quench during the training of some magnets. These "cleaning" cycles improve in some cases the beginning of the training. Figure 1 shows the best example. This cleaning process often changed the nature of the quench such that they were not preceeded by measurable spikes.

However similar evidences are observed for different ramp conditions.

- A similar improvement was observed when going directly to quench at a rate of $40 \mathrm{~A} / \mathrm{s}$ instead of the usual $2 \mathrm{~A}$ s.

- The ramp-rate dependence of the quench level is drastically improved by current precycles [8].

It is therefore believed that the non-uniform distribution of the current between the strands of the cable plays a role in the level of the training quenches. Magnetic measurements show that these inhomogeneities of current sharing change during time intervals equivalent to those of these tests and strongly depend on cycling conditions [5]. Quenches occured at currents near to the conductor limit whilst the winding is at constant current for a few minutes without any change in temperature.

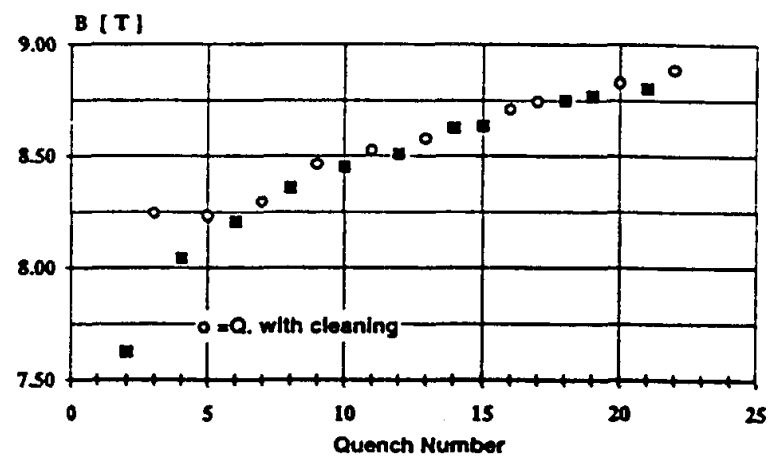

Fig. 1. Training improvement due to "cleaning" cycles on every second quench on the MTAIE magnet.

\section{Forced Training}

Natural quenches or transitions provoked by energy extraction dissipate similar amounts of energy in the conductor and give rise to the same hot spot temperature estimated from the measured MIITS [9]. Figure 2 shows that provoking quenches at current levels just below the expected level of the next training quench has a similar effect as a natural training quench. The MTAlA magnet was known to have a slow and regular training. Quenches provoked at much lower current values are not improving the training.

This induces two important consequences:

- abrupt heating of the winding appearing when the level of forces is still high release weak points that would otherwise generate training quenches,

- a region detected to be weak by the quench location techniques could hide a slightly less weak region.

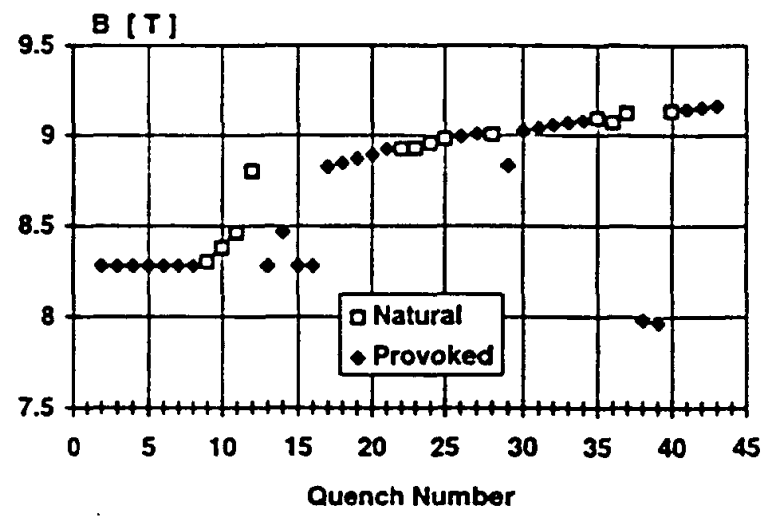

Fig. 2. Forced training of the MTAlA magnet. Provoked quenches are induced by fast decay of the current that heats up the full winding through intersurand coupling currents. 


\section{Training Rate for Different Types of Magnets}

Quench performances and training rates are similar between single aperture and twin aperture magnets. This was also found for $10 \mathrm{~m}$ long models. The performances, therefore, do not depend on the number or length of the coils tested. That complements the information obtained by forced training.

\section{E. Modifications Pefformed on some Magnets}

Several $1 \mathrm{~m}$ long models were modified either to vary the compression from the iron yoke to the coils collars or to improve the shimming between the collars and the windings. With the exceptions listed below, these modifications either are neutral or slightly degrade the training performances. Disassembling the collars usually provokes a degradation. Increasing the longitudinal compression of the ends was also tried without success.

Weak improvements are barely noticeable when the collaryoke interference is increased to have the iron yoke still open in cold conditions. These improvements are observed for magnets clearly having their training quenches at the ends of the windings.

The MTAlA magnet has shown a clear improvement after full disassembly, exchange of spacers of the ends of the windings, and partial impregnation of the ends.

The MTAlH remained far from its conductor limit without the iron yoke structure compressing the collars and then afterwards when completely assembled it almost reached the limit.

\section{F. Thermal Cycles to Room Temperature}

After a thermal cycle to room temperature, the first quench level is clearly higher and the number of quenches needed to reach the current level previously obtained is much smaller. However, some magnets have their first quench at a similar value to the one of the first quench of the first training if they stay for weeks at room temperature. The improvement of the rate of the subsequent training is nevertheless preserved.

\section{G. Unstable Training Performances}

The MTA3C magnet has reached its short sample limit of $10.5 \mathrm{~T}$ at $2.13 \mathrm{~K}$. Following tests without extracting part of the stored energy into an external dump resistor have degraded the performance of one outer layer, possibly due to a very high prestress. The hot spot temperature reached was below $230 \mathrm{~K}$. Tentative repairs have not allowed this field record to be reached again. It is however still the magnet that reaches the highest field after a period at room temperature $(8.85 \mathrm{~T})$.

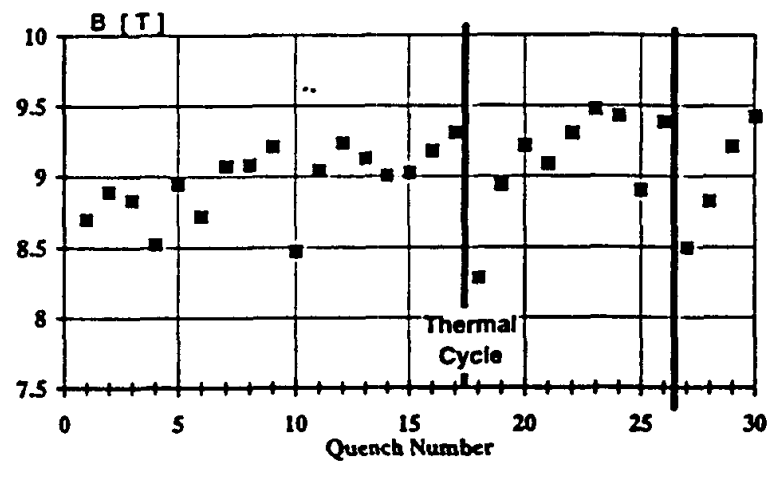

Fig. 3. Training curve of the $10 \mathrm{~m}$ long MTP1A3 magnet.

Small retrainings have been observed on a short magnet after having made quenches without energy extraction. Unstable training performances are also seen on several other magnets (Fig. $3 \& 4$ ).

It is therefore postulated that, although the abrupt heating of the conductor during a quench may induce improvements in the training, it can as well degrade the training.

\section{BEHAVIOUR OF THE MBTRA}

The MBTRA was wound with the $12 \mathrm{~mm}$ wide SSC cable. It has therefore a lower conductor limit of $9.65 \mathrm{~T}$ at $2.0 \mathrm{~K}$. The field levels of the training (Fig. 4) are nevertheless similar to those of most of the magnets wound with a $17 \mathrm{~mm}$ wide cable.

The density of magnetic energy dissipated in the conductor is however bigger than for the $17 \mathrm{~mm}$ cable magnets. The hot spot temperature reached a maximum of $370 \mathrm{~K}$ in the outer layer when the energy extracted to a dump resistor was reduced to $55 \%$. The magnet did not show any degradation after this quench.

This MBTRA magnet was instrumented with spot heaters located between the first turn of the inner layer and the end key spacer. Voltage taps were installed on each side of the spot heaters in order to measure the resistive voltage growth

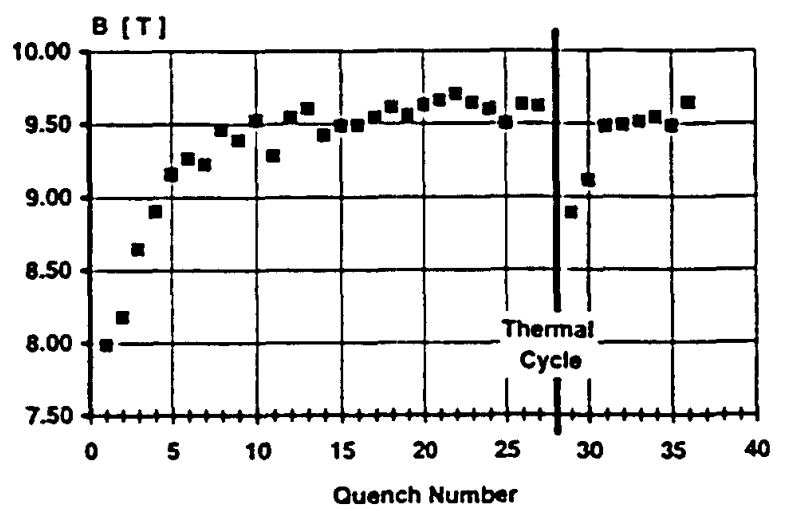

Fig.4. Trining curve of the MBTRA magne. 
TABLE II

MAXIMUM TEMPERATURES REACHED ON THE MBTRA MAGNET. COMPARISON BETWEEN THE MITTS AND VALUES DEDUCED FROM THE MEASURED RESISTANCE GROWTH DURING A QUENCH [10]

\begin{tabular}{ccccc}
\hline $\begin{array}{c}\text { Current } \\
\text { [A] }\end{array}$ & $\begin{array}{c}\text { Local Field } \\
\text { [T] }\end{array}$ & $\begin{array}{c}\text { MIITs } \\
\text { [MA^2.s] }\end{array}$ & $\begin{array}{c}\text { Temperarure deduced from } \\
\text { [K] } \\
\text { MIITS }\end{array}$ & \begin{tabular}{c} 
Resistance \\
\hline
\end{tabular} \\
\hline 4146 & 3.23 & 4.88 & 55 & 51 \\
6226 & 4.85 & 5.68 & 67 & 64 \\
7782 & 6.06 & 8.59 & 105 & 91 \\
9341 & 7.27 & 9.74 & 130 & 126 \\
9999 & 7.79 & 10.25 & 142 & 135 \\
\hline
\end{tabular}

in the cable once the quench was triggered by one of these heaters. This resistance allows the maximum temperature to be deduced. Table II shows, for quenches triggered at different current levels, the comparison between these maximum temperatures and the ones deduced by the time integral of the current squared (MIITs). The adiabatic calculations were made under the assumptions that only $5 \%$ of the cable insulation warms up to the same temperature as the conductor. The magneto-resistance effect is calculated from Kohler's plot [11].

\section{LESSONS FROM THE TRAININGS PERFORMED}

- The $10 \mathrm{~m}$ long magnets tested have training performances similar to those of both the single aperture and twin aperture short models. The weak points detected in the windings are measured to be the same.

- Some magnets have their first quench at a similar value to the one of the first quench of the first training if they stay for weeks at room temperature.

- The structure of the ends of the windings and of the clamping elements around the ends and the splice-layer jump transition must be improved. Several developments were started and new versions of coils will be tested next autumn.

- Detailed mechanical design of the support of the cables in the coils is more important than the temperature margin of the conductor with regard to training performances.

- Quenches dissipating high densities of energy in the conductor sometimes induce unstable behaviour in this type of magnet having unprecedented high force levels.

\section{Conclusions}

The recent lest of four $10 \mathrm{~m}$ long, $50 \mathrm{~mm}$ aperture dipoles have proven the usefulness of an extensive short model programme to steer the design and the execution of the final $14 \mathrm{~m}$ long. $56 \mathrm{~mm}$ aperture dipole magnets needed for the LHC accelerator. The training behaviour is similar for full length magnets and both single and twin aperture short models. The weak points in the structure are found to be the same. Premature quenches are located in the first turns of mainly the outer layer in the ends of the windings. Numerous quenches were also located at the transitions between different collar shapes in the "layer jump/splice" length, where the cable of the inner layer ramps and is soldered to the outer layer cable.

Short sample measurements of both single strands anri cables predict accurately the conductor limits of the magnets at both $4.3 \mathrm{~K}$ and $2 \mathrm{~K}$.

Good agreement is found for the maximum temperature of the conductor during a quench calculated either from the measured MIITs or from the measured resistance growth.

The windings working at stress levels corresponding to the limit of the creep of the insulation have a poor memory of a previous training after a period at room temperature. It is therefore important to study and correct the weak points that are responsible for the first quenches of the training to get a reasonable working margin with respect to the $8.36 \mathrm{~T}$ specified for the LHC.

For these reasons, CERN has launched an extensive program of single aperture short models based on the new parameters of the LHC dipoles. One important goal is to improve the windings in the ends. The first single aperture models based on the new design will be tested in autumn 1995 , followed soon after by twin aperture models.

\section{ACKNOWLEDGMENT}

The constant support and the devotion of the whole test station operation team and of the cryogenic operators were key elements for the success of the numerous tests quoted.

\section{REFERENCES}

[1] The LHC Study Group. "Large Hadron Collider Accelerator Project" (Revised June 1995). CERN/AC95-05(LHC). June 1995.

[2] R. Perin. "Starus of the LHC program and magnet development". proc. of Applied Superconductivity Conference, 1994, in press.

(3) A. Yamamolo at al.. "Development of twin aperture dipole magnets for the large hadron collider", proc. of Applied Superconductivity Conference. 1994, in press.

[4] J. Ostler a al." Design. Fabrication and lesting of a $56 \mathrm{~mm}$ Bore TwinAperture $1 \mathrm{~m}$ long Dipole Magnet made with SSC type cable". CERN, Geneva, CH, to be presented at this conference.

[5] J. Buckley ex al. "Dynamic magnetic measurements of superconducting magnets for the LHC proc. of Applied Superconductivity Conference. 1994. in press.

[6] A. Siemko et al.. "Quench location in the superconducting model magnets for the LHC by means of pick-up coils", proc. of Applied Superconductivity Conference. 1994, in press.

[7] D. Leroy et al. "Test results on 10 T LHC superconducting one meter long dipole models ". IEEE uans. of applied supereonductivity, vol. 3. No I. march 1993.

[8] A. Verweij et al. " Ramp Rate induced Quenches for the 1 meter dipole model magnets for the CERN LHC., proc. of Applied Superconductivity Conference, 1994, in press.

[9] J. Billan ex al. "Test of $1 \mathrm{~m}$ long model magnets for LHC", IEEE trans. on Mag. Vol. 28. 1992. pp. 362-365.

(10) F. Calvone, CERN. Geneva, CH, personnal communication, 1995.

[11] Cryogenic Properties of Copper. Intemational Copper Research Association. 